\title{
Early Perihematomal Edema Expansion: Definition, Significance, and Association with Outcomes after Intracerebral Hemorrhage
}

\author{
Xin-Ni Lv, ${ }^{1}$ Zuo-Qiao Li, ${ }^{1}$ Lan Deng, ${ }^{1}$ Wen-Song Yang, ${ }^{1,2}$ Yu-Lun Li, ${ }^{3}$ Yuan-Jun Huang, \\ Yi-Qing Shen, ${ }^{1,2}$ Xiong-Fei Xie, ${ }^{3}$ Xin-Hui Li, ${ }^{1,2}$ Zi-Jie Wang, ${ }^{1}$ Zhi-Wei Zhang, ${ }^{3}$ Fa-Jin Lv, ${ }^{3}$ \\ Jin-Biao Luo, ${ }^{4}$ Shu-Jie Sun, ${ }^{5}$ Peng Xie, ${ }^{1,2}$ and Qi Li $\mathbb{1}^{1}$ \\ ${ }^{1}$ Department of Neurology, The First Affiliated Hospital of Chongqing Medical University, Chongqing 400016, China \\ ${ }^{2}$ NHC Key Laboratory of Diagnosis and Treatment on Brain Functional Diseases, The First Affiliated Hospital of Chongqing \\ Medical University, Chongqing 400016, China \\ ${ }^{3}$ Department of Radiology, The First Affiliated Hospital of Chongqing Medical University, Chongqing 400016, China \\ ${ }^{4}$ Department of Neurosurgery, Guangzhou First People's Hospital, School of Medicine, South China University of Technology, \\ Guangzhou 510180, China \\ ${ }^{5}$ Department of Neurosurgery, The Xuhui Hospital Affiliated to Fudan University, Shanghai 200031, China
}

Correspondence should be addressed to Qi Li; qili_md@126.com

Received 12 April 2021; Revised 29 August 2021; Accepted 31 August 2021; Published 13 September 2021

Academic Editor: Mayur Parmar

Copyright (C) 2021 Xin-Ni Lv et al. This is an open access article distributed under the Creative Commons Attribution License, which permits unrestricted use, distribution, and reproduction in any medium, provided the original work is properly cited.

Objective. To investigate the association between early perihematomal edema (PHE) expansion and functional outcome in patients with intracerebral hemorrhage (ICH). Methods. Patients with ICH who underwent initial computed tomography (CT) scans within 6 hours after the onset of symptoms and follow-up CT scans within $24 \pm 12$ hours were included. Absolute PHE increase was defined as the absolute increase in PHE volume from baseline to 24 hours. A receiver-operating characteristic (ROC) curve was generated to determine the cutoff value for early PHE expansion, which was operationally defined as an absolute increase in PHE volume of $>6 \mathrm{~mL}$. The outcome of interest was 3-month poor outcome defined as modified Rankin scale score of $\geq 4$. A multivariable logistic regression procedure was used to assess the association between early PHE expansion and outcome after ICH. Results. In 233 patients with ICH, 89 (38.2\%) patients had poor outcome at 3-month follow-up. Early PHE expansion was observed in 56 of 233 (24.0\%) patients. Patients with early PHE expansion were more likely to have poor functional outcome than those without $(43.8 \%$ vs. $11.8 \%, p<0.001)$. After adjusting for age, admission systolic blood pressure, admission Glasgow Coma Scale score, baseline ICH volume and the presence of intraventricular hemorrhage, and time from onset to CT, early PHE expansion was associated with poor outcome (adjusted odds ratio, 4.25 ; $95 \%$ confidence interval, 1.70 10.60; $p=0.002$ ). Conclusions. The early PHE expansion was not uncommon in patients with ICH and was correlated with poor outcome following ICH.

\section{Introduction}

Spontaneous intracerebral hemorrhage (ICH) is a lifethreatening subtype of stroke with high morbidity and disability $[1,2]$. Age, admission Glasgow Coma Scale (GCS) score, baseline hematoma size, hemorrhage location, and the presence of intraventricular hemorrhage (IVH) have been well established as predictors of functional outcome after $\mathrm{ICH}$, according to pre- vious studies [3-5]. Perihematomal edema (PHE) is generally considered as a radiological marker for secondary injury after ICH [6]. Several studies have investigated the relationship between PHE volume and outcome after $\mathrm{ICH}$, and the results have been inconsistent [7-10]. Some studies found that PHE volume predicted short-term and long-term outcomes in patients with ICH $[7,9]$. Recent studies reported no significant association between PHE and functional outcome $[8,10]$. Few 
studies have focused on the dynamic change in PHE. We aimed to investigate whether early PHE expansion within the first 36 hours could be associated with poor outcome in patients with $\mathrm{ICH}$.

\section{Subjects and Methods}

2.1. Study Design. Our study included ICH patients admitted between July 2011 and July 2017 in the First Affiliated Hospital of Chongqing Medical University from our ongoing prospective cohort database. All participants or their legal surrogates were provided written informed consent. This study was conducted in accordance with local ethical frameworks. We recruited ICH patients who had undergone an initial computed tomography (CT) scan within 6 hours after symptoms and a follow-up CT scan within $24 \pm 12$ hours. Patients with early surgical intervention before follow-up CT scans and those with secondary ICH were excluded. Demographic and clinical characteristics including age, sex, premorbid medical history of hypertension, diabetes mellitus, heart disease, renal disease, smoking status, and premorbid modified Rankin scale (mRS) score were collected for each patient. Detailed data such as the admission blood pressure, time between onset of symptoms and baseline CT scan, and the GCS score were prospectively collected.

2.2. Imaging Analysis. We saved Digital Imaging and Communications in Medicine (DICOM) format data of both admission and follow-up CT scans which were collected for further review. $\mathrm{ICH}$ and $\mathrm{PHE}$ volumes on admission and follow-up CT scans were segmented by a semiautomated computer-assisted volumetric analysis as previously described (Version 11.0; AnalyzeDirect, Overland Park, KS, USA) [11]. In brief, the PHE volume was measured slice by slice on both baseline and follow-up CT scans. All images were evaluated independently by two experienced neurologists in a blinded manner. Hematoma expansion was defined as an increase in hematoma volume of $>33 \%$ or $>6 \mathrm{~mL}$ at follow-up CT scan $[12,13]$. A semiautomated threshold-based approach (range of 5-33 Hounsfield Units) was applied to identify edematous regions as previously described [14]. The discriminant value of the early PHE expansion was determined by the receiveroperating characteristic (ROC) curve analysis. We chose the optimal cutoff that had the highest sensitivity and specificity for defining early PHE expansion. We calculated the sensitivity and specificity for this dichotomous threshold and used multivariable logistic regression to adjust for potential confounding of important risk factors. Multivariate logistic regression analyses were performed by using variables with $p$ $<0.1$ in the univariate analysis. Variables known to be related to poor clinical outcome based on several external datasets were also entered into the multivariable model.

2.3. Outcome Assessment. The functional outcome was assessed using mRS at 3 months after the onset of symptoms by phone interviews (performed by trained physicians). Poor outcome in this study was defined as 3-month mRS of 4-6.

2.4. Statistical Analysis. Data management and analysis were conducted using SPSS software (Version 25.0; IBM Corpora- tion, Armonk, NY, United States). Discrete variables were presented as percentages (\%), and the continuous data were expressed as the mean \pm standard deviation (SD) or median and interquartile range (IQR), as applicable. The baseline variables, including age, sex, blood pressure, and hematoma volume, were compared with Fisher's exact test, Student's $t$ test, or Mann-Whitney $U$ test as appropriate. Using univariate logistic regression analysis, clinical and neuroimaging variables were tested for their association with clinical outcome. Furthermore, we utilized ROC curve analyses to determine the early PHE expansion cutoffs and the related sensitivity and specificity in the prediction of 3-month poor outcome. A multivariable logistic regression procedure was used to assess variables. A $p$ value $<0.05$ was considered statistically significant.

\section{Results}

3.1. Baseline Characteristics. A total of 233 patients were included in our final analysis. The flowchart for inclusion is illustrated in Figure 1. The mean age of the participants was 60.2 years (range, 29.0-94.0 years), and 85 patients (36.5\%) were women. The median time from symptom onset to the baseline CT scan was 2.0 hours (IQR, 1.0-4.0). The median baseline ICH volume was $13.4 \mathrm{~mL}$ (IQR, 8.8-21.1 mL), and the median baseline PHE volume was $6.2 \mathrm{~mL}$ (IQR, 3.2$10.5 \mathrm{~mL})$. Intraventricular hemorrhage was observed in 75 of 233 (32.2\%) patients on the initial CT scan. In our study population, the baseline hematoma was located in the basal ganglia $(n=138,59.2 \%)$, thalamus $(n=62,26.6 \%)$, and others $(n=33$, $14.2 \%)$. Patients with poor outcome were more likely to have a larger absolute PHE increase (median 1.5 vs. $3.8 \mathrm{~mL}, p<0.001$ ). Significant early PHE expansion was present in 56 of 233 (24.0\%) patients on follow-up CT scan. The baseline demographic and clinical characteristics are described in Table 1.

3.2. Receiver-Operating Characteristic Analysis. Univariate ROC analysis revealed a high diagnostic performance for early PHE expansion in the discrimination of good versus poor outcome after ICH with an optimal cutoff of 6.83 (area under the curve, AUC 0.704, 41.6\% sensitivity, 90.3\% specificity). To facilitate clinical evaluation, we considered a value of $6 \mathrm{~mL}$ as the optimal threshold for early PHE expansion which was determined by ROC curve analyses. Early PHE expansion $>6$ $\mathrm{mL}$ was observed in $56(24.0 \%)$ patients. The sensitivity and specificity value of PHE expansion $>6 \mathrm{~mL}$ for predicting poor outcome at 3 months were $43.8 \%$ and $88.2 \%$, respectively.

3.3. Predictors of Functional Outcome in ICH Patients. A total of 89 (38.2\%) patients had poor outcome (mRS 4-6) at 3-month follow-up (Table 2). After adjusting for age, admission systolic blood pressure, admission GCS score, baseline hematoma volume and the presence of intraventricular hemorrhage, and time from onset to CT, early PHE expansion was associated with poor outcome (adjusted odds ratio (OR), 4.25; 95\% confidence interval (CI), 1.70-10.60; $p=0.002$ ) (Table 2). The distribution of 3-month $\mathrm{mRS}$ in patients with and without early PHE expansion is shown in Figure 2. 


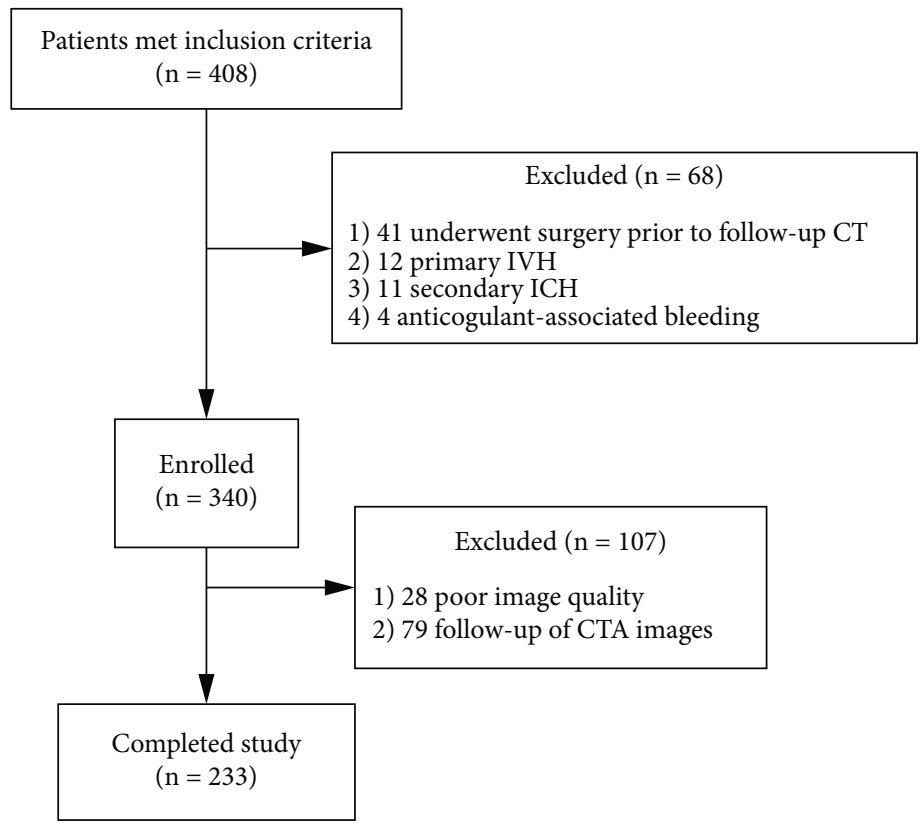

FIGURE 1: Cohort selection flowchart. IVH: intraventricular hemorrhage; ICH: intracerebral hemorrhage.

3.4. Factors Associated with PHE Expansion. Univariate and multivariate logistic regression analyses were performed to assess the association between various parameters and early PHE expansion (Table 3). In the univariate analysis, the admission systolic blood pressure, admission GCS score, baseline hematoma volume, and time from onset to CT were associated with PHE expansion $>6 \mathrm{~mL}$ (all $p$ values $<0.1$ ). In multivariable logistic regression analysis, systolic blood pressure (OR, 1.02; 95\% CI, 1.00-1.03; $p=0.022$ ), baseline hematoma volume (OR, 1.11; 95\% CI, 1.07-1.15; $p<0.001$ ), and the time from onset to CT (OR, 0.59; 95\% CI, 0.44$0.78 ; p<0.001)$ remained independent predictors of early PHE expansion.

\section{Discussion}

We demonstrated that an absolute PHE increase correlated with the 3-month clinical outcome in ICH patients. Moreover, our selected dichotomous threshold, early PHE expansion $>6$ $\mathrm{mL}$, was strongly associated with poor clinical outcome after adjusting for potential confounding factors.

Previous reports investigated the association between PHE increase and outcome when analyzing PHE evolution up to 24 or 72 hours in ICH patients [15-17]. A pooled analysis of the Intensive Blood Pressure Reduction in Acute Cerebral Haemorrhage Trial (INTERACT) 1 and 2 trials showed that early absolute PHE increase up to 24 hours after onset was associated with increased 90-day odds of death or dependency [15]. A recent analysis of $\mathrm{ICH}$ patients from the Virtual International Stroke Trials Archive-ICH (VISTA-ICH) study $(n=596)$ demonstrated that 72-hour absolute PHE volume growth was independently associated with poor outcome in the basal ganglia ICH patients or those with hematoma volumes $<30$ $\mathrm{mL}$ [16]. A retrospective single-center study found that a $24-$ hour absolute PHE increase was independently associated with mortality and functional outcome in ICH patients [17]. Several studies have investigated the association between relative $\mathrm{PHE}$ (defined as PHE volume/hematoma volume) and functional outcome $[7,8,10,18]$. An early study concluded an inverse correlation between a larger relative edema volume and worse 12 -week functional outcome [10]. However, subsequent studies have failed to identify an association between relative PHE and outcomes $[7,8,18]$. Consistent with previous studies, we also demonstrated that an absolute PHE increase was independently associated with poor outcome. However, there are no well-established criteria for early PHE expansion in patients with ICH. In our study, we proposed a cutoff value for the diagnosis of early PHE expansion on CT based on ROC curves. Early PHE expansion was operationally defined as an increase in its volume by $>6 \mathrm{~mL}$. We found that early PHE expansion occurred in 56 of 233 (24.0\%) ICH patients. Since early PHE expansion is related to poor outcome after $\mathrm{ICH}$, identifying patients with PHE may be useful for designing treatment trials to mitigate early PHE expansion.

Previous studies suggested that hematoma expansion (HE) mostly occurred within 3 hours after the onset of symptoms and was rare after 24 hours $[3,4]$. Unlike early hematoma growth, 2-stage edema PHE expansion has been reported in recent observational studies [19]. The early phase of PHE expansion usually develops as early as 3 hours after the onset of symptoms and increases over time by approximately $100 \%$ during the first 24 hours [15-17]. The PHE expansion rate was defined as the PHE volume increase divided by the time interval between the baseline and the follow-up scans [17]. Several studies have investigated the association between PHE expansion rate and functional outcome in ICH patients $[17,20]$. In a study of 139 patients, Urday et al. reported that a higher 24-hour edema expansion rate after ICH was associated with worse outcome [17]. A recent retrospective analysis showed that the edema expansion 
TABle 1: Comparison of baseline demographic, clinical, and radiological characteristics between patients with and without favorable outcome.

\begin{tabular}{|c|c|c|c|}
\hline \multirow{2}{*}{ Variables } & \multicolumn{3}{|c|}{ Patients, no. (\%) } \\
\hline & Favorable outcome $(n=144,61.8 \%)$ & Poor outcome $(n=89,38.2 \%)$ & $p$ value \\
\hline \multicolumn{4}{|l|}{ Demographic } \\
\hline Mean age, y (SD) & $58.6(11.5)$ & $62.7(12.4)$ & 0.011 \\
\hline Sex, male, $n(\%)$ & $87(60.4)$ & $61(68.5)$ & 0.211 \\
\hline \multicolumn{4}{|l|}{ Medical history } \\
\hline Alcohol consumption, $n(\%)$ & $57(39.6)$ & $38(42.7)$ & 0.638 \\
\hline Smoking, $n(\%)$ & $61(42.4)$ & $44(49.4)$ & 0.291 \\
\hline Hypertension, $n(\%)$ & $103(71.5)$ & $71(79.8)$ & 0.160 \\
\hline Diabetes mellitus, $n(\%)$ & $14(9.7)$ & $12(13.5)$ & 0.376 \\
\hline \multicolumn{4}{|l|}{ Clinical features } \\
\hline Systolic blood pressure, mmHg (SD) & $167.5(26.0)$ & $176.6(35.0)$ & 0.035 \\
\hline Diastolic blood pressure, mmHg (SD) & $97.3(14.5)$ & $101.2(21.6)$ & 0.128 \\
\hline Admission GCS score, median (IQR) & $14(13-15)$ & $10(7-14)$ & $<0.001$ \\
\hline Baseline ICH volume, mL (IQR) & $11.7(7.1-16.4)$ & $18.7(11.1-32.0)$ & $<0.001$ \\
\hline Time from onset to CT, h(IQR) & $2.0(1.0-4.0)$ & $2.0(1.0-3.0)$ & 0.046 \\
\hline Admission PHE volume, mL (IQR) & $5.3(2.6-8.3)$ & $7.5(4.7-14.7)$ & $<0.001$ \\
\hline Follow-up PHE volume, mL (IQR) & $7.8(4.0-11.1)$ & $13.7(7.0-25.1)$ & $<0.001$ \\
\hline Absolute PHE increase, mL (IQR) & $1.5(0.1-3.5)$ & $3.8(1.2-11.9)$ & $<0.001$ \\
\hline Early PHE expansion, $n(\%)$ & $17(11.8)$ & $39(43.8)$ & $<0.001$ \\
\hline SAH at baseline CT, $n(\%)$ & $11(7.6)$ & $9(10.1)$ & 0.513 \\
\hline IVH at baseline CT, $n(\%)$ & $28(19.4)$ & $47(52.8)$ & $<0.001$ \\
\hline Hematoma growth, $n(\%)$ & $27(18.8)$ & $45(50.6)$ & $<0.001$ \\
\hline \multicolumn{4}{|l|}{ ICH location } \\
\hline Basal ganglia hemorrhage, $n(\%)$ & $95(66.0)$ & $43(48.3)$ & 0.008 \\
\hline Thalamic hemorrhage, $n(\%)$ & $29(20.1)$ & $33(37.1)$ & 0.004 \\
\hline Other, $n(\%)$ & $20(13.9)$ & $13(14.6)$ & 0.879 \\
\hline \multicolumn{4}{|l|}{ Outcome } \\
\hline In-hospital mortality, $n(\%)$ & $0(0)$ & $16(18.0)$ & $<0.001$ \\
\hline 30-day mortality, $n(\%)$ & $0(0)$ & $33(37.1)$ & $<0.001$ \\
\hline 90-day mRS, median (IQR) & $1(0-2)$ & $6(4.5-6)$ & $<0.001$ \\
\hline
\end{tabular}

ICH: intracerebral hemorrhage; CT: computed tomography; IVH: intraventricular hemorrhage; SAH: subarachnoid hemorrhage; GCS: Glasgow Coma Scale; mRS: modified Rankin scale; PHE: perihematomal edema; IQR: interquartile range; and SD: standard deviation.

rate over the first 24 hours correlated with death or disability for both lobar and deep spontaneous ICH. However, the 72hour PHE expansion rate was associated with worse functional outcome only in deep ICH patients [20]. In addition, late-phase PHE growth occurs between 2 and 4 weeks after the ICH event. Animal and in vitro studies have suggested that the development of brain edema is activated by an increase in the production of proinflammatory and anti-inflammatory mediators within hours after stroke $[21,22]$. This correlation between the time from symptom onset to CT and PHE evolution in the acute process was confirmed in our study. Therefore, we have used an early timeframe for PHE assessment to capture PHE expansion. Recent analysis of the Antihypertensive Treatment of Acute Cerebral Hemorrhage-2 (ATACH-2) study suggested that ultraearly ( $<2$ hours after onset) blood pressure reduction was associated with reduced $\mathrm{HE}$ and improved outcome in ICH patients [23]. Therapy focusing on reducing $\mathrm{HE}$ or $\mathrm{PHE}$ expansion may be effective within the early therapeutic time-window. In our study, we observed that $24.0 \%$ of patients experienced PHE expansion within 6 hours after the onset of symptoms. We also demonstrated that early PHE expansion was associated with poor outcome in ICH patients. Since early PHE expansion may be a promising target for intervention, early identification of $\mathrm{PHE}$ and initiation of antiedema expansion treatment may be useful in the management of $\mathrm{ICH}$ patients.

In patients with $\mathrm{ICH}$, previous studies defined hematoma growth as a $33 \%$ increase in hematoma volume or $>6 \mathrm{~mL}$ at follow-up CT scan in previous studies $[12,13]$. Recently, Yogendrakumar et al. proposed revised criteria for defining $\mathrm{HE}$ in the ICH populations [24]. However, there are no wellestablished criteria for defining PHE expansion. In previous studies, an absolute PHE volume increase was associated with poor outcome in patients with ICH. In the present study, we proposed a quantitative criterion for PHE expansion in the early phase. Therefore, strategies targeting this early phase of 
TABLE 2: Univariate and multivariate analysis of predictors for poor outcome (mRS 4-6) at 3 months.

\begin{tabular}{|c|c|c|c|}
\hline Variable & Odds ratio & 95\% confidence interval & $p$ value \\
\hline \multicolumn{4}{|l|}{ Univariate analysis } \\
\hline Age, year ${ }^{\mathrm{a}}$ & 1.03 & $1.01-1.05$ & 0.012 \\
\hline Sex, male & 1.43 & $0.82-2.50$ & 0.212 \\
\hline Alcohol consumption & 1.14 & $0.67-1.95$ & 0.639 \\
\hline Smoking & 1.33 & $0.78-2.26$ & 0.292 \\
\hline Hypertension & 1.57 & $0.84-2.95$ & 0.161 \\
\hline Diabetes mellitus & 1.45 & $0.64-3.29$ & 0.378 \\
\hline Systolic blood pressure, $\mathrm{mmHg}^{\mathrm{a}}$ & 1.01 & $1.00-1.02$ & 0.025 \\
\hline Diastolic blood pressure, $\mathrm{mmHg}^{\mathrm{a}}$ & 1.01 & $1.00-1.03$ & 0.097 \\
\hline Admission GCS score ${ }^{\mathrm{a}}$ & 0.69 & $0.62-0.77$ & $<0.001$ \\
\hline Baseline $\mathrm{ICH}$ volume, $\mathrm{mL}^{\mathrm{a}}$ & 1.07 & $1.04-1.10$ & $<0.001$ \\
\hline IVH at baseline CT & 4.64 & $2.58-8.33$ & $<0.001$ \\
\hline Time from onset to CT, hour ${ }^{\mathrm{a}}$ & 0.84 & $0.71-1.00$ & 0.046 \\
\hline Admission PHE volume, $\mathrm{mL}^{\mathrm{a}}$ & 1.12 & $1.06-1.17$ & $<0.001$ \\
\hline Absolute PHE increase, $\mathrm{mL}^{\mathrm{a}}$ & 1.16 & $1.09-1.23$ & $<0.001$ \\
\hline Early PHE expansion & 5.83 & $3.02-11.24$ & $<0.001$ \\
\hline \multicolumn{4}{|l|}{ Multivariate analysis } \\
\hline Age, year ${ }^{\mathrm{a}}$ & 1.03 & $1.00-1.06$ & 0.073 \\
\hline Systolic blood pressure, $\mathrm{mmHg}^{\mathrm{a}}$ & 1.01 & $0.99-1.02$ & 0.289 \\
\hline Admission GCS score ${ }^{\mathrm{a}}$ & 0.76 & $0.68-0.86$ & $<0.001$ \\
\hline Baseline ICH volume, $\mathrm{mL}^{\mathrm{a}}$ & 1.06 & $1.02-1.11$ & 0.003 \\
\hline IVH at baseline CT & 5.41 & $2.48-11.81$ & $<0.001$ \\
\hline Time from onset to CT, hour ${ }^{\mathrm{a}}$ & 0.98 & $0.78-1.24$ & 0.852 \\
\hline Early PHE expansion & 4.25 & $1.70-10.60$ & 0.002 \\
\hline
\end{tabular}

ICH: intracerebral hemorrhage; CT: computed tomography; IVH: intraventricular hemorrhage; GCS: Glasgow Coma Scale; mRS: modified Rankin scale; PHE: perihematomal edema; OR: odds ratio; CI: confidence interval. ${ }^{a}$ Per unit change in regressor.

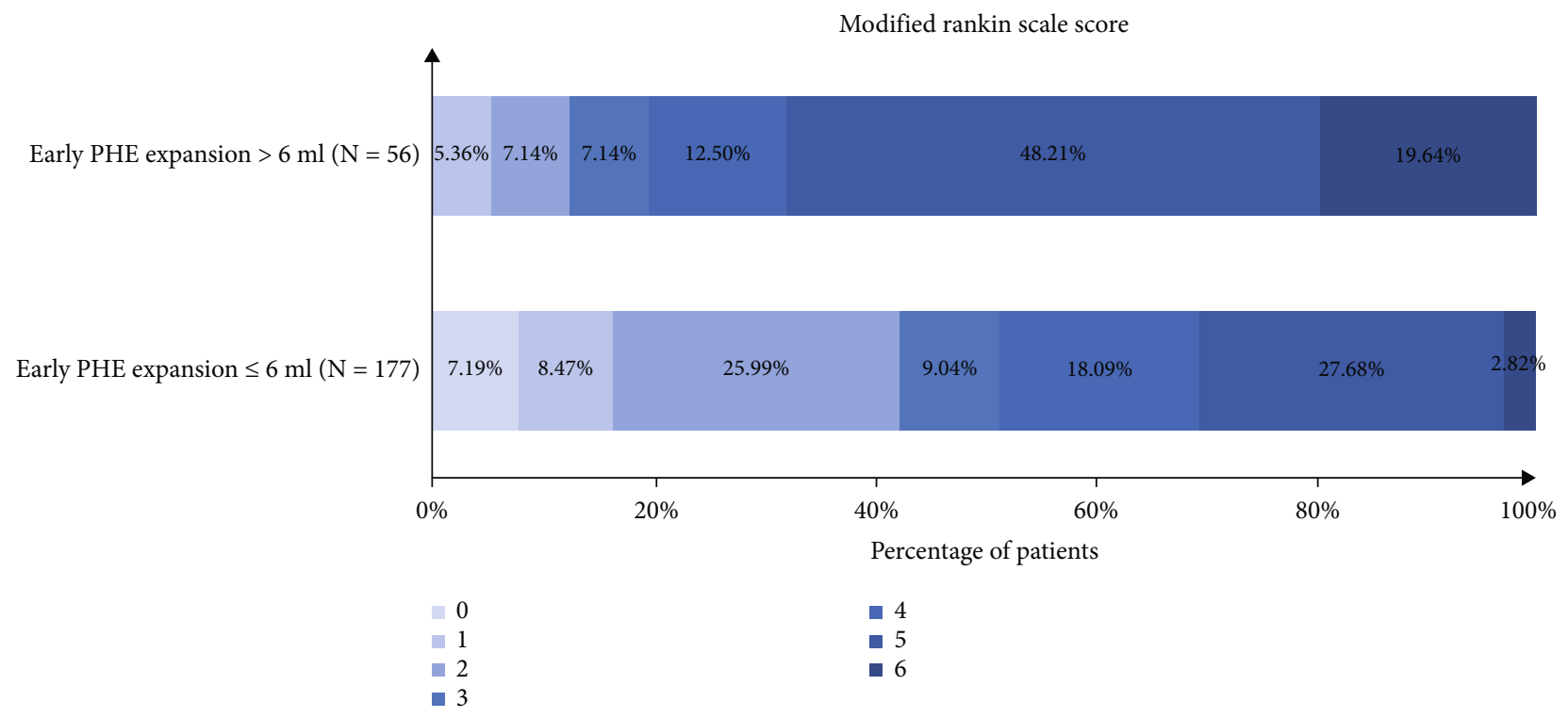

FIgURe 2: Distribution of modified Rankin scale score in patients with or without early PHE expansion. PHE: perihematomal edema. 
TABLE 3: Univariate and multivariate analysis of predictors for early PHE expansion.

\begin{tabular}{|c|c|c|c|}
\hline Variable & Odds ratio & 95\% confidence interval & $p$ value \\
\hline \multicolumn{4}{|l|}{ Univariate analysis } \\
\hline Age, year ${ }^{\mathrm{a}}$ & 1.00 & $0.97-1.02$ & 0.839 \\
\hline Sex, male & 1.60 & $0.83-3.07$ & 0.160 \\
\hline Alcohol consumption & 1.49 & $0.82-2.73$ & 0.195 \\
\hline Smoking & 1.73 & $0.94-3.16$ & 0.077 \\
\hline Hypertension & 1.02 & $0.51-1.96$ & 0.949 \\
\hline Diabetes mellitus & 1.19 & $0.47-2.99$ & 0.715 \\
\hline Systolic blood pressure, $\mathrm{mmHg}^{\mathrm{a}}$ & 1.02 & $1.01-1.03$ & 0.005 \\
\hline Admission GCS score $^{\mathrm{a}}$ & 0.86 & $0.78-0.93$ & $<0.001$ \\
\hline Baseline $\mathrm{ICH}$ volume, $\mathrm{mL}^{\mathrm{a}}$ & 1.10 & $1.06-1.13$ & $<0.001$ \\
\hline IVH at baseline CT & 0.89 & $0.47-1.71$ & 0.736 \\
\hline Time from onset to CT, hour ${ }^{\mathrm{a}}$ & 0.66 & $0.52-0.83$ & $<0.001$ \\
\hline \multicolumn{4}{|l|}{ Multivariate analysis } \\
\hline Systolic blood pressure, $\mathrm{mmHg}^{\mathrm{a}}$ & 1.02 & $1.00-1.03$ & 0.022 \\
\hline Admission GCS score ${ }^{\mathrm{a}}$ & 0.95 & $0.85-1.06$ & 0.388 \\
\hline Baseline $\mathrm{ICH}$ volume, $\mathrm{mL}^{\mathrm{a}}$ & 1.11 & $1.07-1.15$ & $<0.001$ \\
\hline Time from onset to CT, hour ${ }^{\mathrm{a}}$ & 0.59 & $0.44-0.78$ & $<0.001$ \\
\hline
\end{tabular}

ICH: intracerebral hemorrhage; CT: computed tomography; IVH: intraventricular hemorrhage; GCS: Glasgow Coma Scale; PHE: perihematomal edema; OR: odds ratio; CI: confidence interval. ${ }^{\text {a }}$ Per unit change in regressor.

PHE expansion are likely to alter the edema growth trajectory with reduced peak edema and may result in improved outcome.

In our study, we also observed that baseline hematoma volume was independently associated with early PHE expansion. From a pathophysiological standpoint, the components of the hematoma may influence PHE evolution [25-28]. Studies have also indicated that the surrogate markers of iron load such as the serum ferritin level and hematocrit are considered to be the relevant factors for edema formation in ICH [25-27]. After erythrocyte lysis, the iron concentration in the brain could reach as high as $10 \mathrm{mmol} / \mathrm{L}$, causing the thrombin-induced brain edema [25]. A recent study showed that a higher admission hematocrit was associated with a greater delay in peak PHE [28]. Theoretically, a larger hematoma size produces a more absolute PHE increase [29]. These findings may indicate a dose effect of blood components and breakdown products on PHE formation.

Our study has several limitations. First, only patients from one single center were included. Second, our study only investigated the dynamic PHE change during the acute phase of ICH. However, PHE eveolution is a biphasic event, and the late-phase PHE changes are still unknown. Future studies with larger sample sizes and follow-up scans at different time points are needed to further clarify the natural history of PHE evolution.

\section{Conclusions}

In summary, we have demonstrated that an absolute PHE increase independently predicted poor outcome in patients with ICH. Early PHE expansion $>6 \mathrm{~mL}$ is an optimal threshold associated with poor outcome in patients with spontaneous ICH within 6 hours of onset. Early PHE expansion may aid in the selection of new therapeutic strategies and patient groups most likely to benefit from intervention. Future studies are needed to determine the effect of anti-PHE expansion treatment in limiting PHE growth.

\section{Data Availability}

The datasets presented in this article are not readily available because the datasets generated for this study are available on request to the corresponding author.

\section{Ethical Approval}

The studies involving human participants were reviewed and approved by the Ethics Committee of The First Affiliated Hospital of Chongqing Medical University.

\section{Conflicts of Interest}

The authors declare no competing interests.

\section{Authors' Contributions}

QL and $\mathrm{X}-\mathrm{NL}$ are responsible for the study concept and design; QL, X-NL, Z-QL, DL, W-SY, Y-LL, Y-JH, Y-QS, XFX, X-HL, Z-JW, and Z-WZ for the acquisition of data; XNL and Z-QL for the statistical analysis; all authors for the analysis and interpretation of data; X-NL for drafting of the manuscript; QL and X-NL for the critical revision of the manuscript for important intellectual content; and QL for the obtained funding and study supervision. Xin-Ni Lv and Zuo-Qiao Li contributed equally to this work. 


\section{Acknowledgments}

This study was supported by grants from the National Key R\&D Program of China (Nos. 2018YFC1312200 and 2018YFC1312203), the National Natural Science Foundation of China (No. 82071337), the Chongqing High-End Young Investigator Project (No. 2019GDRC005), and the Science and Technology Innovation Project of "ChengduChongqing Economic Circle” (No. KJCXZD2020019).

\section{References}

[1] A. I. Qureshi, A. D. Mendelow, and D. F. Hanley, "Intracerebral haemorrhage," Lancet, vol. 373, no. 9675, pp. 16321644, 2009.

[2] C. J. van Asch, M. J. Luitse, G. J. Rinkel, I. van der Tweel, A. Algra, and C. J. M. Klijn, "Incidence, case fatality, and functional outcome of intracerebral haemorrhage over time, according to age, sex, and ethnic origin: a systematic review and metaanalysis," Lancet Neurology, vol. 9, no. 2, pp. 167-176, 2010.

[3] H. B. Brouwers, Y. Chang, G. J. Falcone et al., "Predicting hematoma expansion after primary intracerebral hemorrhage," JAMA Neurology, vol. 71, no. 2, pp. 158-164, 2014.

[4] C. Delcourt, Y. Huang, H. Arima et al., "Hematoma growth and outcomes in intracerebral hemorrhage: the INTERACT1 study," Neurology, vol. 79, no. 4, pp. 314-319, 2012.

[5] Q. Li, R. Li, L. B. Zhao et al., "Intraventricular hemorrhage growth: definition, prevalence and association with hematoma expansion and prognosis," Neurocritical Care, vol. 33, no. 3, pp. 732-739, 2020.

[6] W. C. Ziai, "Hematology and inflammatory signaling of intracerebral hemorrhage," Stroke, vol. 44, 6, Supplement 1, pp. S74-S78, 2013.

[7] G. Appelboom, S. S. Bruce, Z. L. Hickman et al., "Volumedependent effect of perihaematomal oedema on outcome for spontaneous intracerebral haemorrhages," Journal of Neurology, Neurosurgery, and Psychiatry, vol. 84, no. 5, pp. 488493, 2013.

[8] B. Volbers, W. Willfarth, J. B. Kuramatsu et al., "Impact of perihemorrhagic edema on short-term outcome after intracerebral hemorrhage," Neurocritical Care, vol. 24, no. 3, pp. 404-412, 2016.

[9] L. H. Sansing, E. A. Kaznatcheeva, C. J. Perkins, E. Komaroff, F. B. Gutman, and G. C. Newman, "Edema after intracerebral hemorrhage: correlations with coagulation parameters and treatment," Journal of Neurosurgery, vol. 98, no. 5, pp. 985992, 2003.

[10] J. M. Gebel Jr., E. C. Jauch, T. G. Brott et al., "Relative edema volume is a predictor of outcome in patients with hyperacute spontaneous intracerebral hemorrhage," Stroke, vol. 33, no. 11, pp. 2636-2641, 2002.

[11] S. Urday, L. A. Beslow, D. W. Goldstein et al., "Measurement of perihematomal edema in intracerebral hemorrhage," Stroke, vol. 46, no. 4, pp. 1116-1119, 2015.

[12] A. M. Demchuk, D. Dowlatshahi, D. Rodriguez-Luna et al., "Prediction of haematoma growth and outcome in patients with intracerebral haemorrhage using the CT-angiography spot sign (PREDICT): a prospective observational study," Lancet Neurology, vol. 11, no. 4, pp. 307-314, 2012.

[13] D. Dowlatshahi, A. M. Demchuk, M. L. Flaherty et al., "Defining hematoma expansion in intracerebral hemorrhage: rela- tionship with patient outcomes," Neurology, vol. 76, no. 14, pp. 1238-1244, 2011.

[14] B. Volbers, D. Staykov, I. Wagner et al., "Semi-automatic volumetric assessment of perihemorrhagic edema with computed tomography," European Journal of Neurology, vol. 18, no. 11, pp. 1323-1328, 2011.

[15] J. Yang, H. Arima, G. Wu et al., "Prognostic significance of perihematomal edema in acute intracerebral hemorrhage: pooled analysis from the intensive blood pressure reduction in acute cerebral hemorrhage trial studies," Stroke, vol. 46, no. 4, pp. 1009-1013, 2015.

[16] S. B. Murthy, Y. Moradiya, J. Dawson et al., "Perihematomal edema and functional outcomes in intracerebral Hemorrhage," Stroke, vol. 46, no. 11, pp. 3088-3092, 2015.

[17] S. Urday, L. A. Beslow, F. Dai et al., "Rate of perihematomal edema expansion predicts outcome after intracerebral hemorrhage," Critical Care Medicine, vol. 44, no. 4, pp. 790-797, 2016.

[18] H. Arima, J. G. Wang, Y. Huang et al., "Significance of perihematomal edema in acute intracerebral hemorrhage: the INTERACT trial," Neurology, vol. 73, no. 23, pp. 1963-1968, 2009.

[19] T. Y. Wu, G. Sharma, D. Strbian et al., "Natural history of perihematomal edema and impact on outcome after intracerebral hemorrhage," Stroke, vol. 48, no. 4, pp. 873-879, 2017.

[20] Z. Grunwald, L. A. Beslow, S. Urday et al., "Perihematomal edema expansion rates and patient outcomes in deep and lobar intracerebral hemorrhage," Neurocritical Care, vol. 26, no. 2, pp. 205-212, 2017.

[21] S. Urday, W. T. Kimberly, L. A. Beslow et al., "Targeting secondary injury in intracerebral haemorrhage-perihaematomal oedema," Nature Reviews. Neurology, vol. 11, no. 2, pp. 111122, 2015.

[22] D. A. Wilkinson, A. S. Pandey, B. G. Thompson, R. F. Keep, Y. Hua, and G. Xi, "Injury mechanisms in acute intracerebral hemorrhage," Neuropharmacology, vol. 134, pp. 240-248, 2018.

[23] Q. Li, A. D. Warren, A. I. Qureshi et al., "Ultra-early blood pressure reduction attenuates hematoma growth and improves outcome in intracerebral hemorrhage," Annals of Neurology, vol. 88, no. 2, pp. 388-395, 2020.

[24] V. Yogendrakumar, T. Ramsay, D. A. Fergusson et al., "Redefining hematoma expansion with the inclusion of intraventricular hemorrhage growth," Stroke, vol. 51, no. 4, pp. 1120-1127, 2020.

[25] F. Huang, G. Xi, R. F. Keep, Y. Hua, A. Nemoianu, and J. T. Hoff, "Brain edema after experimental intracerebral hemorrhage: role of hemoglobin degradation products," Journal of Neurosurgery, vol. 96, no. 2, pp. 287-293, 2002.

[26] M. Lou, K. Lieb, and M. Selim, "The relationship between hematoma iron content and perihematoma edema: an MRI study," Cerebrovascular Diseases, vol. 27, no. 3, pp. 266-271, 2009.

[27] M. Mehdiratta, S. Kumar, D. Hackney, G. Schlaug, and M. Selim, "Association between serum ferritin level and perihematoma edema volume in patients with spontaneous intracerebral hemorrhage," Stroke, vol. 39, no. 4, pp. 1165-1170, 2008.

[28] C. Venkatasubramanian, M. Mlynash, A. Finley-Caulfield et al., "Natural history of perihematomal edema after intracerebral hemorrhage measured by serial magnetic resonance imaging," Stroke, vol. 42, no. 1, pp. 73-80, 2011.

[29] D. Staykov, I. Wagner, B. Volbers et al., "Natural course of perihemorrhagic edema after intracerebral hemorrhage," Stroke, vol. 42, no. 9, pp. 2625-2629, 2011. 